\title{
Adjacent Crossings Do Matter
}

\author{
Radoslav Fulek $^{1, \star}$, Michael J. Pelsmajer ${ }^{2, \star \star}$, \\ Marcus Schaefer ${ }^{3}$, and Daniel Štefankovič ${ }^{4}$ \\ 1 Ecole Polytechnique Fédérale de Lausanne, Lausanne, Switzerland \\ radoslav.fulek@epfl.ch \\ 2 Illinois Institute of Technology, Chicago, IL 60616, USA \\ pelsmajer@iit.edu \\ 3 DePaul University, Chicago, IL 60604, USA \\ mschaefer@cs.depaul.edu \\ 4 University of Rochester, Rochester, NY 14627, USA \\ stefanko@cs.rochester.edu
}

\begin{abstract}
In a drawing of a graph, two edges form an odd pair if they cross each other an odd number of times. A pair of edges is independent if they share no endpoint. For a graph $G$, let $\operatorname{ocr}(G)$ be the smallest number of odd pairs in a drawing of $G$ and let $\operatorname{iocr}(G)$ be the smallest number of independent odd pairs in a drawing of $G$. We construct a graph $G$ with $\operatorname{iocr}(G)<\operatorname{ocr}(G)$, answering a question by Székely, and-for the first time - giving evidence that crossings of adjacent edges may not always be trivial to eliminate.

The graph $G$ is based on a separation of iocr and ocr for monotone drawings of ordered graphs. A drawing of a graph is $x$-monotone if every edge intersects every vertical line at most once and every vertical line contains at most one vertex. A graph is ordered if each of its vertices is assigned a distinct $x$-coordinate. We construct a family of ordered graphs such that for $x$-monotone drawings, the monotone variants of ocr and iocr satisfy mon-iocr $(G)<O\left(\operatorname{mon}-\operatorname{ocr}(G)^{1 / 2}\right)$.
\end{abstract}

\section{Introduction}

When drawing a graph some assumptions are natural: there are only finitely many crossings, no more than two edges cross in a point, edges do not pass through vertices, and edges do not touch 1 Sometimes these assumptions are relaxed (degenerate drawings allow more than two edges to cross in a point), and sometimes more restrictions are added, for example adjacent edges may not be allowed to cross.

The crossing number $\operatorname{cr}(G)$ of a graph $G$ is the smallest number of crossings in a drawing of $G$. It is easy to see that in an optimal drawing, adjacent edges

\footnotetext{
* The first author gratefully acknowledges support from the Swiss National Science Foundation Grant No. 200021-125287/1.

** The second author gratefully acknowledges the support from NSA Grant H9823008-1-0043 and the Swiss National Science Foundation Grant No. 200021-125287/1.

${ }^{1}$ For a detailed discussion see 14 .
} 
of $G$ do not cross (such crossings can always be removed). This may have led researchers on crossing numbers to think that adjacent crossings are irrelevant or even to prohibit them in drawings 2 Another source for ignoring adjacent crossings may be the fact that graph drawings are often straight-line drawings in which adjacent edges naturally cannot cross.

Pach and Tóth point out in "Which Crossing Number is It Anyway?" that there have been many different ideas on how to define a notion of crossing number, including the following (see [6]14]):

pair crossing number: $\operatorname{pcr}(G)$, the smallest number of pairs of edges crossing in a drawing of $G$,

odd crossing number: $\operatorname{ocr}(G)$, the smallest number of pairs of edges crossing oddly (odd pairs) in a drawing of $G$.

Tutte introduced another type of crossing number by orienting edges arbitrarily, then letting $\lambda(e, f)$ be the difference in the number of crossings where $e$ is pointed to the left of $f$ and the number of crossings where $e$ is pointed to the right of $f$. Changing the orientation of $e$ or $f$ will only change the sign of $\lambda(e, f)$, so one can define:

algebraic crossing number: $\operatorname{acr}(G)$, the minimum of $\sum|\lambda(e, f)|$ in a drawing of $G$, where the sum is taken over pairs of edges $e, f$.

By definition we have ocr $(G) \leq \operatorname{pcr}(G) \leq \operatorname{cr}(G)$ and $\operatorname{ocr}(G) \leq \operatorname{acr}(G) \leq \operatorname{cr}(G)$.

For each of these notions, one can ask whether adjacent crossings matter. In [5], Pach and Tóth suggest a systematic study of this issue (see also [1, Section 9.4]) by introducing two rules: "Rule +" restricts the drawings to drawings in which adjacent edges are not allowed to cross. "Rule -" allows crossings of adjacent edges, but does not count them towards the crossing number. Each parameter ocr, pcr, acr, and cr can be modified by either rule, but since $\mathrm{cr}_{+}=\mathrm{cr}$ (implied by the discussion at the beginning of the section), this yields up to eleven possible distinct variants.

The tables below are based on a figure from [1. The notion of ocr - was introduced as the independent odd crossing number, iocr, by Székeley 14

\begin{tabular}{l||l|l|l} 
Rule + & ocr $_{+}$ & pcr $_{+}$ & \multirow{2}{*}{$\mathrm{cr}$} \\
\hline & ocr & pcr & \\
\hline Rule - & iocr $=$ ocr $_{-}$ & pcr $_{-}$ & cr $_{-}$
\end{tabular}

\begin{tabular}{l||l|l|l} 
& ocr $_{+}$ & acr $_{+}$ & \multirow{2}{*}{$\mathrm{cr}$} \\
\hline & ocr & acr & \\
\hline & iocr $=$ ocr $_{-}$ & acr & (cr \\
\hline
\end{tabular}

It immediately follows from the definitions that the values in each table increase monotonically as one moves from the left to the right and from the bottom to the top. Not much more is known about the relationships between these crossing number variants. In [5], Pach and Tóth write, "We cannot prove anything

\footnotetext{
${ }^{2}$ Székely discusses this issue in 14 .

${ }^{3}$ Székeley credits Tutte [18] with the (implicit) definition of iocr, but Tutte is really concerned with the algebraic crossing numbers only, acr and acr - ; he does not consider parity.
} 
else about iocr $(G)$, pcr $-(G)$, and $\mathrm{cr}_{-}(G)$. We conjecture that these values are very close to $\operatorname{cr}(G)$, if not the same. That is, we believe that by letting pairs of incident edges cross an arbitrary number of times, we cannot effectively reduce the total number of crossings between independent pairs of edges.' 4 Tutte [18 seems to have had a similar opinion, when he explained his choice to study acr writing, "We are taking the view that crossings of adjacent edges are trivial, and easily got rid of." Székely 14 later commented "We interpret this sentence as a philosophical view and not a mathematical claim." West [20] and Székely [15] mention the specific question of whether there are graphs with $\operatorname{iocr}(G)<\operatorname{ocr}(G)$.

There are situations when the entire system of crossing numbers collapses. The classic Hanani-Tutte theorem states that if a graph can be drawn in the plane so that no pair of independent edges crosses an odd number of times, then it is planar [318]. In other words, $\operatorname{iocr}(G)=0$ implies that $\operatorname{cr}(G)=0$ and, thus, that all of the eleven variants are equal (to zero). This was extended to show that all eleven variants are equal as long as $\operatorname{iocr}(G) \leq 2[12$. Székely gave an explicit criterion for when all variants are equal [16. It is also known that all eleven variants are within a square of each other, since $\operatorname{cr}(G) \leq\left(\begin{array}{c}2 \operatorname{iocr}(G) \\ 2\end{array}\right)$ [12. For drawings of $G$ on the projective plane $N_{1}$, we know that $\operatorname{iocr}_{N_{1}}(G)=0$ implies that $\operatorname{cr}_{N_{1}}(G)=0$, so again all variants are equal (to zero) in this case 8 .

Setting aside the Rule - variants, there are some strong results for the remaining seven variants, ocr, ocr $_{+}$, acr, acr $_{+}$, pcr, pcr $_{+}$and $\mathrm{cr}$. If $\operatorname{ocr}(G) \leq 3$ then all these seven variants are equal 9. For drawings on any surface $S$, if $\operatorname{ocr}_{S}(G)=0$ then all seven variants are equal (to zero) [11. Valtr [19] showed that $\operatorname{cr}(G)=O\left(\operatorname{pcr}^{2}(G) / \log \operatorname{pcr}(G)\right)$, which Tóth [17] improved to $\operatorname{cr}(G)=$ $O\left(\operatorname{pcr}^{2}(G) / \log ^{2} \operatorname{pcr}(G)\right)$.

On the other hand, we know that ocr and pcr differ: there is an infinite family of graphs with $\operatorname{ocr}(G)<0.867 \cdot \operatorname{pcr}(G)[10$. Tóth improved this by giving a family of graphs with $\operatorname{acr}(G)<0.855 \cdot \operatorname{pcr}(G)$ [17] $(\operatorname{soocr}(G)<0.855 \cdot \operatorname{pcr}(G)$ as well). For such $G$ it immediately follows that $\operatorname{ocr}(G)<\operatorname{cr}(G)$ and $\operatorname{acr}_{-}(G)<\operatorname{cr}(G)$, answering questions of Pach and Tóth [6] and Tutte [18]; additional consequences can be deduced from the tables above. However, none of these results address the intuitions expressed by Tutte and by Pach and Tóth about how Rule - may or may not affect cr, pcr, ocr, or acr.

We can finally give a result of this nature.

Theorem 1. For every $n$, there is a graph $G$ with $\operatorname{iocr}(G)<\operatorname{ocr}(G)-n$.

In short, adjacent crossings matter 5

To prove Theorem 1, we will first prove a separation for monotone drawings of ordered graphs. An ordered graph is a graph with a total ordering of its vertices. For our purposes, we will assume that the vertex set of an ordered graph is a subset of the integers, and we will only consider drawings where each vertex

\footnotetext{
${ }^{4}$ Some authors write incident edges to mean two edges that share an endpoint, but we will only use adjacent edges. Non-adjacent edges are also called independent edges.

${ }^{5}$ Among other things, Theorem 1 justifies the rather baroque NP-completeness proof for iocr in 13. NP-completeness of ocr is simpler in comparison 7.
} 
$n$ has $x$-coordinate equal to $n$. A drawing of a graph is $x$-monotone if every edge intersects every vertical line at most once and every vertical line contains at most one vertex. We can generalize each crossing number variant to $x$-monotone drawings of ordered graphs $G$, which we denote $\operatorname{mon}-\operatorname{cr}(G), \operatorname{mon}-\operatorname{ocr}(G)$, $\operatorname{mon}-\operatorname{iocr}(G)$, etc.

Pach and Tóth proved that mon-ocr $(G)=0$ implies mon-cr $(G)=0$ [7]. We strengthened this by showing that mon-iocr $(G)=0$ implies $\operatorname{mon}-\operatorname{cr}(G)=0[2$, which had been left as an open problem in [7]. On the other hand, in the same paper we showed that for every $n$ there is a graph $G$ such that $\operatorname{mon}-\operatorname{cr}(G) \geq n$ and $\operatorname{mon} \operatorname{ocr}(G)=1$. In this paper, we will show that there can also be an arbitrary gap between mon-ocr and mon-iocr.

Theorem 2. For every $n \geq 3$ there is an ordered graph $G$ with mon-iocr $(G)=$ $3 n<n^{2}+n=\operatorname{mon}-\operatorname{ocr}(G)$.

Note that for such $G$, we have $\operatorname{mon}-\operatorname{iocr}(G)=O\left(\operatorname{mon}-\operatorname{ocr}(G)^{1 / 2}\right)$. We will use Theorem 2 to prove Theorem 1 .

\section{Separating Monotone Crossing Numbers}

We generalize the crossing number definitions for graphs with weighted edges. Suppose that $G$ is a graph and each edge $e$ has weight $w(e)$. A crossing between edges $e$ and $f$ is assigned crossing weight equal to the product $w(e) w(f)$. Let $D$ be an arbitrary drawing of $G$, and define

$\operatorname{cr}(D)=$ the sum of crossing weights, taken over all crossing in $D$, $\operatorname{ocr}(D)=$ the sum of $w(e) w(f)$, taken over all odd pairs $e, f$ in $D$, $\operatorname{iocr}(D)=$ the sum of $w(e) w(f)$, taken over all independent odd pairs $e, f$ in $D$.

Let $\operatorname{cr}(G)=\min _{D} \operatorname{cr}(D), \operatorname{ocr}(G)=\min _{D} \operatorname{ocr}(D)$, and $\operatorname{iocr}(G)=\min _{D} \operatorname{iocr}(D)$, with each minimum taken over all drawings $D$ of $G$. If we assign every edge weight equal to 1 , then these definitions revert back to their original, unweighted versions.

Consider an ordered graph $G=([7],\{13,16,23,24,25,27,35,37,46,47,56\})$ with edge weights $w(16)=w(23)=w(25)=w(27)=w(46)=w(47)=2 x^{2}$, $w(24)=w(37)=x, w(13)=w(35)=w(56)=1$ (see the left of Figure 1 for a drawing of $G$ ).

Theorem 3. For the weighted ordered graph $G$ in Figure 1 with $x \geq 3$, we have

$$
\operatorname{mon}-\operatorname{iocr}(G)=3 x<x^{2}+x=\operatorname{mon}-\operatorname{ocr}(G) .
$$

Proof. In the drawing on the left side of Figure 1, the only independent pairs of edges that cross oddly are $(13,24),(24,35),(56,37)$, showing

$$
\operatorname{mon}-\operatorname{iocr}(G) \leq 3 x \text {. }
$$



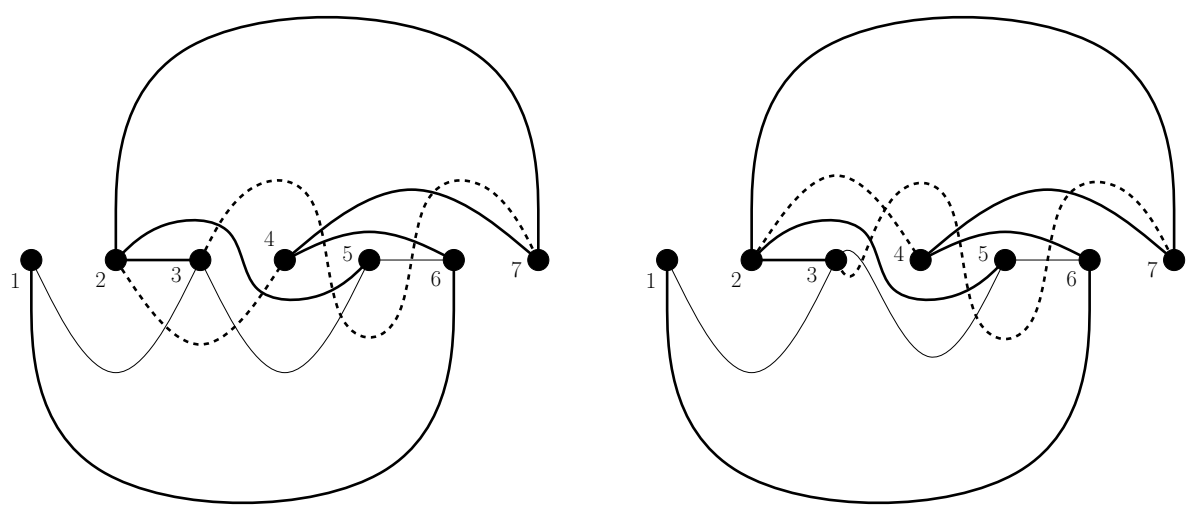

Fig. 1. Two drawings of a weighted ordered graph $G$ with mon-iocr $(G)<\operatorname{mon-ocr}(G)$; thick solid edges have weight $2 x^{2}$, the thick dashed edges have weight $x$, and the thin solid edges have weight 1 . The left drawing shows that mon-iocr $(G) \leq 3 x$; the right drawing that mon-ocr $(G) \leq x^{2}+x$.

The drawing also shows mon-ocr $(G) \leq 4 x+2 x^{3}$, since for mon-ocr the odd pairs $(24,25)$ and $(35,37)$ count. If we reroute edge 24 to go above 25 , it crosses 37 (instead of 13,35 , and 25). Close to 3 , we can twist 35 and 37 so they cross evenly. This yields the drawing on the right in Figure 1, It shows that

$$
\operatorname{mon}-\operatorname{ocr}(G) \leq x^{2}+x \text {. }
$$

Suppose that we have a drawing $D$ of $G$ with mon-iocr $(D)<x^{2}+x$. Since $x^{2}+x<2 x^{2}$ for $x \geq 3$, no thick edge (that is an edge of weight $2 x^{2}$ ) is crossed oddly by an independent edge. We claim that this forces most of the drawing to be as depicted in Figure 1. Without loss of generality assume that 46 passes above 5. Then 35 must pass below 4 (to avoid crossing 46 oddly) and 47 must pass above 5 and 6 (to avoid crossing 35 and 56 oddly). Now 16 has to pass below 4 (to avoid 47) and hence below 2,3,5 (to avoid 24, 23, 35). Since 16 goes below 2 we have that 27 is above 6 (to avoid 16) and also above 3,4,5 (to avoid $56,35,46$ ). Then 13 has to be below 2 (to avoid 27) and 25 has to be above 3 (to avoid 13) and below 4 (to avoid 46). The edge 37 has to go below 5 (to avoid 25) above 6 (to avoid 16) and hence above 4 (to avoid crossing 46 oddly). Note that we have determined the above-below relationship for all relevant edge-vertex pairs (when the vertex lies between the endpoints of the edge) except for those with the edge 24, using only the fact that thick edges cannot be crossed oddly by independent edges. Note that thus far, $(37,56)$ is the only independent odd pair of edges.

Consider how the edge 24 can be drawn. If we draw it above 3 then it will cross 37 oddly bringing the total number of odd crossings between independent pairs of edges to $x^{2}+x$. Thus 24 has to go below 3. To summarize: we have shown that any drawing $D$ of $G$ with mon-iocr $(D)<x^{2}+x$ must have the same (or mirrored) above-below relationships as in the drawing on Figure 1. Note that 
24 crosses 13 and 35 oddly, bringing the total number of odd crossings between independent pairs of edges to $3 x$. This proves the left equality in (1).

We next prove the right equality in (1). For this we need only show $\operatorname{mon}-\operatorname{ocr}(G) \geq x^{2}+x$, due to (3). Suppose that we have a drawing $D$ of $G$ such that mon-ocr $(D)<x^{2}+x$. This implies mon-iocr $(D)<x^{2}+x$ so by the earlier argument we may assume that every relevant edge-vertex pair has the same above-below relationship in $D$ as in the drawing of Figure 1 .

If 24 leaves 2 above 23 then 23 and 24 cross oddly (since 24 goes below 3 ) showing mon-ocr $(D) \geq 2 x^{3}+3 x$, a contradiction. Thus 24 leaves 2 below 23 . If 24 leaves 2 below 25 then 24 and 25 cross oddly (since 25 goes below 4 ) showing mon-ocr $(D) \geq 2 x^{3}+3 x$, a contradiction. Thus 24 leaves 2 above 25 . Now, using transitivity, 23 leaves 2 above 25 but that means that 23 and 25 cross oddly (since 25 goes above 3 ) showing mon-ocr $(D) \geq 2 x^{2}+3 x$, a contradiction. Hence there is no drawing $D$ of $G$ with mon-ocr $(D)<x^{2}+x$, finishing the proof of (1).

\subsection{From Weighted Edges to Unweighted Edges}

Suppose that $G$ is a graph or ordered graph with edges of positive integer weight. Let $G^{\prime}$ be the graph obtained by replacing each edge of weight $w$ with $w$ edges of weight 1 , equivalently, with $w$ unweighted edges. Choose any of the eleven crossing variants mentioned in Section 1] and consider a drawing of $G^{\prime}$ (which is $x$-monotone if $G$ is an ordered graph) that optimizes that crossing variant. Suppose that $e_{1}$ and $e_{2}$ are copies of the same edge $e$ of $G$. Without loss of generality, we may assume that $e_{1}$ contributes less than or equal to what $e_{2}$ contributes to the chosen crossing parameter. We can redraw $e_{2}$ along the side of $e_{1}$ so that they do not cross; then $e_{2}$ will contribute the same to the crossing parameter as $e_{1}$, so the new drawing is still optimal. Hence, we may assume that in an optimal drawing of $G^{\prime}$, multiple edges are drawn in a bundle, all with essentially the same behavior 6 It follows that all crossing parameters are the same for $G$ and $G^{\prime}$.

Lemma 1. Subdividing an edge of a graph does not change ocr or iocr. Subdividing an edge of an ordered graph near one of its endpoints does not change mon-ocr or mon-iocr. These results hold for graphs with multiple edges as well.

Proof. Let $G$ be a graph or ordered graph, possibly with multiple edges. If $G$ is an ordered graph, we will restrict all drawings to be $x$-monotone drawings.

Fix an ocr-optimal (iocr-optimal) drawing of $G$, and choose any edge $u v$. Subdivide $u v$ with a vertex $z$, which is added to the drawing of $u v$ near the endpoint $u$. Then for each edge $e \neq u v$, $e$ will cross $z v$ oddly if and only if $e$ crossed $u v$ oddly, and $e$ does not cross $u z$ at all. Hence ocr is unchanged; iocr is also unchanged unless $e$ shares an endpoint with $u v$ but not with $z v$, which means that $e$ is incident to $u$ but not $v$. In this case, we can deform a small section of $e$ until it passes over $z$ (while maintaining its monotonicity, if $G$ is

${ }^{6}$ This argument was probably first made in Kainen 4] for the standard crossing number. 
ordered); do this for all such $e$. This yields a drawing with iocr no bigger than in the initial drawing.

Now consider any drawing of the new graph. We can erase $z$ from that drawing to obtain a drawing of the original, unsubdivided graph. If $G$ is ordered, then we erase $z$ from an $x$-monotone drawing where $z$ lies strictly between $u$ and $v$, so we obtain an $x$-monotone drawing of $G$. Erasing $z$ moves all odd pairs of edges with $u z$ or $z v$ to become odd pairs with $u v$ (and if an edge crosses both $u z$ and $z v$ oddly, then these cancel and it crosses $u v$ evenly). Hence the number of odd pairs and independent odd pairs does not increase.

Consider any integer $x \geq 3$. Replace the weighted edges of the graph in Figure 1 by multiple edges, and then apply Lemma 1 to every edge. We obtain an unweighted ordered graph $H$ with mon-iocr $(H)=3 x<x^{2}+x=\operatorname{mon-ocr}(H)$. Thus, Theorem 2 is proved.

Before moving on, note that for any drawing of a graph $G$, we can remove self-intersections of edges without adding any crossing or odd pair, by redrawing locally near the crossing as shown in Figure 2] (originally from [10]).
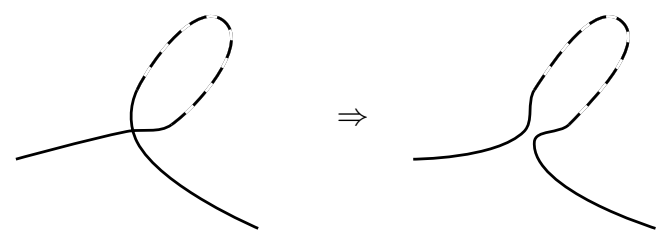

Fig. 2. Removing a self-intersection

\section{Adjacent Crossings Are Not Trivial}

Given an ordered graph $G=(V, E)$ with $V=\left\{v_{1}<v_{2} \cdots<v_{n}\right\}$ let $G^{\prime}$ be obtained from $G$ by adding the following framework: start with a cycle $C_{2 n+2}$ formed from two paths $s, u_{1}, \ldots, u_{n}, t$ and $s, w_{1}, \ldots, w_{n}, t$; call this the outer framework. Add paths $Q_{i}=u_{i} v_{i} w_{i}$ for $1 \leq i \leq n$; call this the inner framework. Assign a weight of $w_{I}=n^{4}+1$ to the edges in the inner framework and a weight of $w_{O}=n^{4}+n^{3} w_{I}+1$ to the edges in the outer framework. Edges originally in $G$ remain at weight 1 (unweighted). From the weighted graph $G^{\prime}$ we will obtain the unweighted graph $G^{\prime \prime}$ by replacing each edge of weight $w>1$ in $G^{\prime}$ by $w$ copies of $P_{3}$.

Lemma 2. With $G^{\prime}$ as defined above we have $\psi\left(G^{\prime \prime}\right)=\operatorname{mon}-\psi(G)+c$ for any connected graph $G$, where $\psi$ is one of the crossing numbers \{iocr, ocr, cr\} and $c=w_{I} \sum_{v_{i} v_{j} \in E(G), i<j}(j-i-1)$.

Lemma 2 and Theorem 2 immediately yield Theorem 1 , In 2 we showed that for every $n$ there is an ordered graph $G$ such that $\operatorname{mon}-\operatorname{cr}(G) \geq n$ and $\operatorname{mon}-\operatorname{ocr}(G)=$ 1. Together with Lemma 2, this yields a new graph $G^{\prime}$ with $\operatorname{ocr}\left(G^{\prime}\right)<\operatorname{cr}\left(G^{\prime}\right)$, 
joining the earlier examples from [10 and 17. In the journal version of this paper, we show that Lemma 2 can be made to work for other crossing numbers as well, so that it has the potential to lead to further separations.

For the proof of Lemma 2, we need the following lemma. An even edge is an edge that crosses every other edge an even number of times (possibly zero times).

Lemma 3 (Pelsmajer, Schaefer, and Štefankovič [9]). If $D$ is a drawing of $G$ in the plane and $E$ is the set of even edges in $D$, then $G$ has a redrawing in which all edges in $E$ are crossing-free, there are no new pairs of edges that cross an odd number of times, and the cyclic order of edges at each vertex does not change.

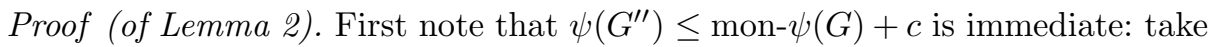
a monotone drawing realizing mon- $\psi(G)$ and overlay it with a planar drawing of the framework, call the resulting drawing $D^{\prime}$ (see Figure 3 for an example). Then $\psi\left(D^{\prime}\right)=$ mon- $\psi(G)+c$ since the only crossings are single crossings between pairs of non-adjacent edges that count the same whatever $\psi$ is. From $D^{\prime}$ we can obtain a drawing $D^{\prime \prime}$ of $G^{\prime \prime}$ by replacing the weighted edges in the drawing by parallel $P_{3} \mathrm{~s}$; then $\psi\left(D^{\prime \prime}\right)=\psi\left(D^{\prime}\right)$ (since the framework edges are not involved in any adjacent crossings), so $\psi\left(G^{\prime \prime}\right) \leq \psi\left(D^{\prime \prime}\right)=\operatorname{mon}-\psi(G)+c$.

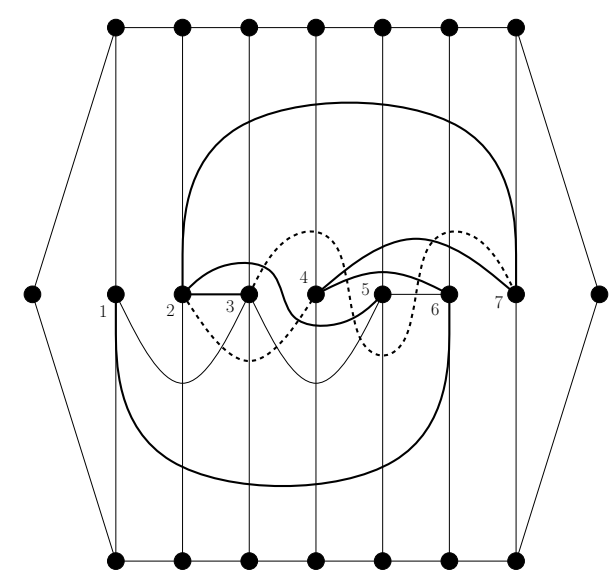

Fig. 3. Overlay of $G$ from Figure 1 with framework (note that in the construction, $G$ will be an unweighted graph)

It remains to prove $\psi\left(G^{\prime \prime}\right) \geq \operatorname{mon}-\psi(G)+c$ for $\psi \in\{\mathrm{cr}$, ocr, iocr $\}$. It is easy to see that $\psi\left(G^{\prime \prime}\right) \geq \psi\left(G^{\prime}\right)$ : fix an $\psi$-optimal drawing of $G^{\prime \prime}$. Consider $w$ parallel paths $P_{3}$ that were used to replace an edge of weight $w$ in $G^{\prime}$. Pick one of these paths $P$ that contributes the smallest amount to $\psi\left(G^{\prime \prime}\right)$. Now redraw the remaining $w-1$ paths to run very close to $P$ and without crossing each other. This redrawing cannot increase the value of $\psi$ of the drawing. But now we can 
bundle the parallel paths into a single weighted edge to obtain a drawing $D^{\prime}$ of $G^{\prime}$ with $\psi\left(D^{\prime}\right) \leq \psi\left(G^{\prime \prime}\right)$. So $\psi\left(G^{\prime}\right) \leq \psi\left(G^{\prime \prime}\right)$.

Hence, to establish the lemma it is sufficient to show that $\psi\left(G^{\prime}\right) \geq \operatorname{mon}-\psi(G)+$ $c$. We proceed in three steps; we first show that there is a $\psi$-minimal drawing of $G^{\prime}$ in which the edges of the outer framework are crossing-free. In the second step we show that we can assume that the edges of the inner framework do not cross each other. In the third step we show that from such a drawing of $G^{\prime}$, we can construct a monotone drawing of $G$ with at most $\psi\left(G^{\prime}\right)-c$ crossings. It follows that mon- $\psi(G) \leq \psi\left(G^{\prime}\right)-c$.

For the first step, fix an $\psi$-minimal drawing of $G^{\prime}$. For $\psi=$ cr the claim is immediate: any edge crossing an edge of the outer framework contributes at least $w_{O}$ to $\psi\left(G^{\prime}\right)$. However, we already proved that $\psi\left(G^{\prime}\right) \leq \operatorname{mon}-\psi(G)+$ $c \leq n^{4}+n^{3} w_{I}<w_{O}$, so all edges of the outer framework must be crossingfree. If $\psi=$ ocr then edges of the outer framework cannot be involved in any odd pairs, since any such odd pair would contribute $w_{O}$ to ocr and, as above, $\psi\left(G^{\prime}\right) \leq \operatorname{mon}-\psi(G)+c \leq n^{4}+n^{3} w_{I}<w_{O}$. So all the edges in the outer framework are even. We can then apply Lemma 3 to make all edges in the outer framework crossing-free without introducing any new pair of edges crossing oddly (in particular, $\psi$ does not increase). This leaves the case $\psi=$ iocr. The argument here is similar to ocr. In any iocr-minimal drawing, edges of the outer framework cannot be involved in any independent odd pairs, so all odd pairs involving these edges must have adjacent edges. However, all vertices in the outer framework have degree 2 or 3 , so we can modify the drawing near each of these vertices to ensure that all the edges in the outer framework are actually even. We then proceed as in the case of ocr.

This completes the first step: we know that we can assume that the outer framework is entirely free of crossings. Since we assumed that $G$ is a connected graph, all vertices of $G$ must lie in the same face of $C_{2 n+2}$, without loss of generality, the inner face. Since every edge not in the outer framework is incident to a vertex of $G$ this also implies that all edges lie in the inner face and the outer face is therefore empty.

In the second step we show that we can assume that edges of the inner framework do not cross each other. Recall that $Q_{i}=u_{i} v_{i} w_{i}$ is the inner framework path passing through $v_{i}$ with endpoints $u_{i}$ and $w_{i}$ on $C_{2 n+2}$, for $1 \leq i \leq n$.

For $\psi=$ cr the claim is immediate again, since any such crossing would contribute $w_{I}^{2}=w_{I}\left(n^{4}+1\right)=n^{4} w_{I}+w_{I}>n^{3} w_{I}+n^{4}+1=w_{O}$ to $\psi\left(G^{\prime}\right)$, but we already know that $\psi\left(G^{\prime}\right) \leq w_{O}$.

For $\psi=$ ocr, we can similarly conclude that any two edges of the inner framework cross evenly, and for $\psi=$ iocr, we know that any independent pair of edges in the inner framework crosses evenly. Suppose that $\psi=$ iocr and two adjacent edges of the inner framework, $u_{i} v_{i}$ and $v_{i} w_{i}$, cross oddly. In that case, we perform a $\left(u_{i} v_{i}, v_{i}\right)$-move (that is, we deform a small section of $u_{i} v_{i}$, bring it close to $v_{i}$ and then make it pass over $\left.v_{i}\right)$; this does not affect iocr and ensures that $u_{i} v_{i}$ and $v_{i} w_{i}$ cross evenly. We conclude that for $\psi \in\{$ ocr, iocr $\}$ any two edges 
of the inner framework cross an even number of times. We next show how to remove crossings between edges of the inner framework.

To this end, let us consider $Q_{1}=u_{1} v_{1} w_{1}$. Let $e$ be an edge of the inner framework that crosses $u_{1} v_{1}$ (we allow the case $e=v_{1} w_{1}$ ). Deform $e$ near each such crossing so that it follows along $u_{1} v_{1}$ toward $v_{1}$ and then over $v_{1}$. Since $e$ must have crossed $u_{1} v_{1}$ an even number of times, this procedure will not change the value of $\psi$ for the drawing. Performing this for all such edges $e$ of the inner framework leaves $u_{1} v_{1}$ free of crossings with edges of the inner framework. This redrawing process may have introduced self-crossings of $v_{1} w_{1}$ which can be removed without affecting $\psi$, as described at the end of Section 2 , So $u_{1} v_{1}$ crosses no edge of the inner framework and $v_{1} w_{1}$ crosses every other edge of the inner framework evenly. Without loss of generality, we can assume that $t$ is in the exterior of $s u_{1} v_{1} w_{1} s$. Then the interior of $s u_{1} v_{1} w_{1} s$ does not contain any vertices: every vertex (other than $t$ ) has a path consisting of edges of weight at least $w_{I}$ to $t$, contributing at least $w_{I}^{2}$ to $\psi$, which we know to be impossible. Now cut each edge $e$ of the inner framework where it crosses $v_{1} w_{1}$. We can partition the crossings of $e$ and $v_{1} w_{1}$ into pairs since they cross evenly, and then for each pair we add curves that run along each side of $v_{1} w_{1}$ that connect the severed ends of $e$. Thus, $e$ is replaced by a curve that may have more than one component, all but one of which are closed curves with no vertex, and none of the components intersect $v_{1} w_{1}$. Because of the way the connecting curves are added in pairs, the value of $\psi$ is unchanged. The components lying within $s u_{1} v_{1} w_{1} s$ are all closed curves without vertices. Moreover, since there is no vertex within that region, they can be deleted without affecting $\psi$. Any two of the curves on the other side of $Q_{1}$ can be merged by erasing a tiny bit of each curve and adding two parallel curves within the region that join the erased bits of opposite curves, giving a wide berth to all vertices, which ensures that $\psi$ is unchanged. Repeating this process merges all curve components in that region into a single curve, and after removing self-intersections we obtain a valid drawing of $e$ within that region. We can now repeat this argument with $Q_{2}$ and $s u_{1} u_{2} v_{2} w_{2} w_{1} s$, and so on, to establish that none of the $Q_{i}, 1 \leq i \leq n$ have crossings with any edges of the inner framework. This completes the second step.

Hence, for the third step, we can assume that every crossing is between two edges of $G$ or between an edge of $G$ and an edge of the inner framework.

At this point, let us deform the whole drawing so that $C_{2 n+2} \cup\left\{Q_{1}, Q_{n}\right\}-\{s, t\}$ is a rectangle and all the $Q_{i}$ are parallel straight-line segments orthogonal to the outer framework.

For $\psi=$ cr we are nearly done: a $G$-edge $e$ connecting $v_{i}$ to $v_{j}$ must cross all $Q_{k}$ with $i<k<j$, forcing at least $c$ crossings. This leaves $\psi\left(G^{\prime}\right)-c \leq$ mon- $\psi(G) \leq n^{4}<w_{I}$ crossings counting towards $\psi\left(G^{\prime}\right)$. Since a crossing with an edge of the inner framework contributes at least $w_{I}$ to $\psi\left(G^{\prime}\right)$ this accounts for all crossings with edges of the inner framework. So an edge $e=v_{i} v_{j}$ crosses all $Q_{k}$ with $i<k<j$ and no other $Q_{k}$ s. The actual behavior of $e$ between two neighboring $Q_{k} \mathrm{~s}$ is irrelevant and within each such region we can replace $e$ by a straight-line segment connecting its crossings between neighboring $Q_{k} \mathrm{~s}$. 
This does not affect $\psi$ and results in a monotone drawing of $G$ with $\psi\left(G^{\prime}\right)-c$ crossings, proving that mon- $\psi(G) \leq \psi\left(G^{\prime}\right)-c$ which is what we had to prove.

For $\psi \in\{$ ocr, iocr $\}$ we need to do a bit more work. A $G$-edge $e$ connecting $v_{i}$ to $v_{j}$ must cross all $Q_{k}$ with $i<k<j$ oddly. So the crossings of $G$-edges with the inner framework contribute at least $c$ to the value of $\psi$. This leaves at most $\psi\left(G^{\prime}\right)-c \leq$ mon- $\psi(G)<w_{I}$ in $\psi\left(G^{\prime}\right)$ unaccounted for. So there are no nonadjacent odd pairs with edges of the inner framework except those absolutely necessary to connect the endpoints of every edge in $G$. The only case in which odd pairs with inner framework edges can still occur is in the iocr case (where such crossings do not count) if an edge $v_{i} v_{j}, i<j$ crosses an adjacent inner framework edge $\left(u_{i} v_{i}, v_{i} w_{i}, u_{j} v_{j}\right.$, or $\left.v_{j} w_{j}\right)$ oddly. In this case we redraw $v_{i} v_{j}$ near each endpoint (if necessary) so that the ends of $v_{i} v_{j}$ at $v_{i}$ and $v_{j}$ lie between $Q_{i}$ and $Q_{j}$; this does not affect iocr and results in $v_{i} v_{j}$ crossing both $Q_{i}$ and $Q_{j}$ an even number of times. It is possible at this point that $v_{i} v_{j}$ crosses both $u_{k} v_{k}$ and $v_{k} w_{k}$ oddly, where $k \in\{i, j\}$. In that case we perform a $\left(v_{i} v_{j}, v_{k}\right)$-move; this does not affect iocr and ensures that $v_{i} v_{j}$ crosses both $u_{k} v_{k}$ and $v_{k} w_{k}$ evenly.

Thus for $\psi \in\{$ ocr, iocr $\}$ we can now assume that if an edge $e=v_{i} v_{j}$ crosses $u_{k} v_{k}$ or $v_{k} w_{k}$ with $k \leq i$ or $k \geq j$ it must do so evenly. As we did above for the inner framework edges, we push all crossings of $e$ with $u_{k} v_{k}$ along $u_{k} v_{k}$ and over $v_{k}$ to $v_{k} w_{k}$ so that $u_{k} v_{k}$ does not cross $e$ at all; pushing $e$ off $u_{k} v_{k}$ does not affect $\psi$, since $e$ crossed $u_{k} v_{k}$ evenly. For all $k \leq i$ and $k \geq j$ cut $e$ at $v_{k} w_{k}$; pair up crossings of $e$ with $v_{k} w_{k}$ and reconnect severed ends of $e$ on both side of $v_{k} w_{k}$ for all $k \leq i, k \geq j$. Closed components of $e$ between $Q_{i}$ and $Q_{j}$ can be reconnected to the arc-component of $e$ without affecting $\psi$. Every other closed component of $e$ is entirely contained in a region which does not contain a vertex, so all such components are even and can be dropped without affecting $\psi$. In the end, all of $e$ lies in the region formed by $C_{2 n+2}$ and $Q_{i}$ and $Q_{j}$.

Now for any $i<k<j$ we have either $\operatorname{ocr}\left(e, u_{k} v_{k}\right)=0$ and $\operatorname{ocr}\left(e, v_{k} w_{k}\right)=w_{I}$ or $\operatorname{ocr}\left(e, u_{k} v_{k}\right)=w_{I}$ and $\operatorname{ocr}\left(e, v_{k} w_{k}\right)=0$ (since we have already accounted for all crossings with edges of weight at least $\left.w_{I}\right)$. For every $k$ push all crossings of $e$ with $Q_{k}$ from the edge with ocr $=0$ to the other edge (not affecting the value of $\psi$ ); that is, $e$ avoids one of the edges of $Q_{k}$ for every $i<k<j$. Let $e^{\prime}$ be any other curve in the region in $C_{2 n+2}$ bounded by $Q_{i}, Q_{j}$ that shares ends with $e$ (here, an end is an endpoint together with a small, crossing-free part of the edge incident to the endpoint); furthermore, suppose that $e^{\prime}$ avoids the same edge in each $Q_{k}$ as does $e$. Then $\operatorname{ocr}(e, g)=\operatorname{ocr}\left(e^{\prime}, g\right)$ for every edge $g$ (other than $e$ ), since $e$ can be continuously deformed to $e^{\prime}$ without passing over any vertex. In particular, we can replace $e$ with a monotone polygonal arc without changing the value of $\psi$. Repeating this for all edges of $G$ gives us a monotone drawing of $G$ with mon- $\psi$ crossings. This completes the argument for $\psi \in\{$ ocr, iocr $\}$.

\section{References}

1. Brass, P., Moser, W., Pach, J.: Research Problems in Discrete Geometry. Springer, New York (2005)

2. Fulek, R., Pelsmajer, M.J., Schaefer, M., Štefankovič, D.: Hanani-Tutte, monotone drawings, and level-planarity. Accepted for WG (2011) 
3. Chojnacki, C., Hanani, H.: Über wesentlich unplättbare Kurven im dreidimensionalen Raume. Fundamenta Mathematicae 23, 135-142 (1934)

4. Kainen, P.C.: A lower bound for crossing numbers of graphs with applications to $K_{n}, K_{p, q}$, and $Q(d)$. J. Combinatorial Theory Ser. B 12, 287-298 (1972)

5. Pach, J., Tóth, G.: Thirteen problems on crossing numbers. Geombinatorics 9(4), 194-207 (2000)

6. Pach, J., Tóth, G.: Which crossing number is it anyway? J. Combin. Theory Ser. B 80(2), 225-246 (2000)

7. Pach, J., Tóth, G.: Monotone drawings of planar graphs. J. Graph Theory 46(1), 39-47 (2004)

8. Pelsmajer, M.J., Schaefer, M., Stasi, D.: Strong Hanani-Tutte on the projective plane. SIAM Journal on Discrete Mathematics 23(3), 1317-1323 (2009)

9. Pelsmajer, M.J., Schaefer, M., Štefankovič, D.: Removing even crossings. J. Combin. Theory Ser. B 97(4), 489-500 (2007)

10. Pelsmajer, M.J., Schaefer, M., Štefankovič, D.: Odd crossing number and crossing number are not the same. Discrete Comput. Geom. 39(1), 442-454 (2008)

11. Pelsmajer, M.J., Schaefer, M., Štefankovič, D.: Removing even crossings on surfaces. European Journal of Combinatorics 30(7), 1704-1717 (2009)

12. Pelsmajer, M.J., Schaefer, M., Štefankovič, D.: Removing independently even crossings. SIAM Journal on Discrete Mathematics 24(2), 379-393 (2010)

13. Pelsmajer, M.J., Schaefer, M., Štefankovič, D.: Crossing numbers of graphs with rotation systems. Algorithmica 60, 679-702 (2011), doi:10.1007/s00453-009-9343-y

14. Székely, L.A.: A successful concept for measuring non-planarity of graphs: the crossing number. Discrete Math. 276(1-3), 331-352 (2004)

15. Székely, L.A.: Progress on Crossing Number Problems. In: Vojtáš, P., Bieliková, M., Charron-Bost, B., Sýkora, O. (eds.) SOFSEM 2005. LNCS, vol. 3381, pp. 53-61. Springer, Heidelberg (2005)

16. Székely, L.A.: An optimality criterion for the crossing number. Ars Math. Contemp. 1(1), 32-37 (2008)

17. Tóth, G.: Note on the pair-crossing number and the odd-crossing number. Discrete Comput. Geom. 39(4), 791-799 (2008)

18. Tutte, W.T.: Toward a theory of crossing numbers. J. Combinatorial Theory 8, 45-53 (1970)

19. Valtr, P.: On the pair-crossing number. In: Combinatorial and Computational Geometry. Math. Sci. Res. Inst. Publ., vol. 52, pp. 569-575. Cambridge University Press, Cambridge (2005)

20. West, D.: Open problems - graph theory and combinatorics, http://www.math.uiuc.edu/ west/openp/ (accessed April 7, 2005) 